\title{
Parallel Operation Program of Marine Power Station Simulation based on Visual C++
}

\author{
Haowen Chen ${ }^{\mathrm{a}}$, Ming Bai ${ }^{\mathrm{b}}$, Rui Zhang, Weishan Li, Runda Lu and Jieqi Zou \\ Guangzhou Maritime Institute, Guangzhou, Guangdong, 510725, China \\ a475178154@qq.com, ${ }^{\mathrm{b}}$ Corresponding author: gzbaiming@163.com
}

\begin{abstract}
Keywords: Marine Power Station; Visual C++;synchronous parallel operation;
Abstract. Automatic synchronous parallel operation is one of main functions of marine power station automation. Taking a semi-submersible vessel as the research object, this paper mainly describes the conditions of synchronous parallel operation, detection and achievement way of synchronous parallel operation. According to the requirement, the marine power station simulation is designed based on the Visual $\mathrm{C}++$ platform. Through experimental validation, the testing results show that the marine power station simulation has a high reliability and good anti -interference.
\end{abstract}

\section{Introduction}

The marine power station generally consists of Marine generator set and switchboard, is the core of marine power system[1].In order to satisfy the power supply reliability and economical efficiency, marine power station usually has three or four synchronous generators as the main power supply. When two or more than two sets of the generators supply to the load, which often requires the generator in parallel operation,and the operation process of Marine generator in parallel operation is called parallel operation.

Now the Marine power station simulator widely applied to crew training , is one kind of combining software with hardware simulator, which plays a huge role in all kinds of crew certificates in field training and competency assessment[2]. But it also has some problems, such as stability improvement, poor extensibility, insufficient secondary development and the location of the training, etc, this influence the training quality and application value of the Marine power station simulator. With the rapid development of computer technology, designing the Marine power station simulatorthrough the Visual $\mathrm{C}++$ platform, which realizes the real time and interactive operation of Marine power station, this has obvious advantages and important significance. It will Improve the quality of teaching and training.

\section{Parallel Operation of The SG(Shaft Generator)s}

\section{The situation of parallel operation}

First, it needs two generators to be operating in parallel when the ship passes in and out the wharf or the narrow waterway in a sailing state. Secondly, the output power of the generator is greater than $80 \%$ of the rated power. Thirdly, the system issues an order of increasing machine when the running generator fault[3].

\section{The parallel conditions required for the parallel connection of SGs}

(1). Voltages should be equal $\left(U_{2}=U_{1}\right.$, The gap between $U_{2}$ and $U_{1}$ permits a figure minus or plus $10 \%$. );

(2). Frequencies should be equal ( $f_{2}=f_{1}$, The gap between $f_{2}$ and $f_{1}$ permits a figure minus or plus $0.5 \mathrm{~Hz}$.);

(3). Parallel connection should be realized at the synchronism time ( $\delta_{2}=\delta_{1}$, The gap between $\delta_{2}$ and $\delta_{1}$ permits a figure minus or plus $15^{\circ}$.);

(4). Phase sequence of the SGs should be same(Because the ship sailing, the line has been connected, it is not necessary to check the phase sequence when parallel operation)[4]; 
The analysis of parallel operation

(1). Fig. 1 show the situation of $f_{2}=f_{1}, \delta_{2}=\delta_{1}, U_{2} \neq U_{1}$,

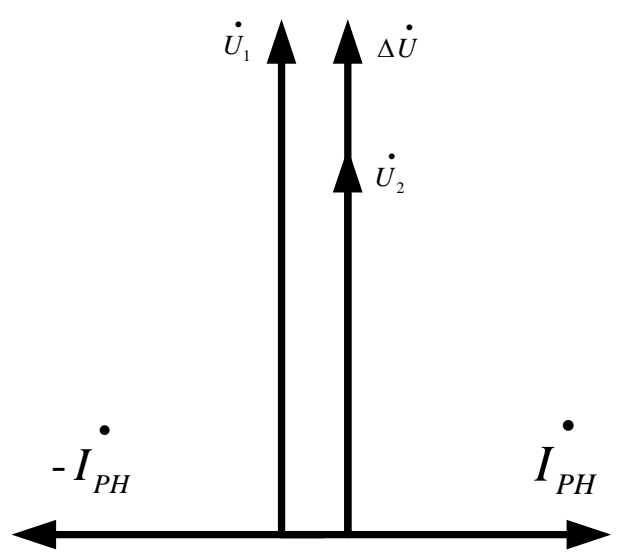

Fig.1 the phasor diagram of $U_{2} \neq U_{1}$

(2). Fig.2 show the situation of $f_{2}=f_{1}, U_{2}=U_{1}, \delta_{2} \neq \delta_{1}$,

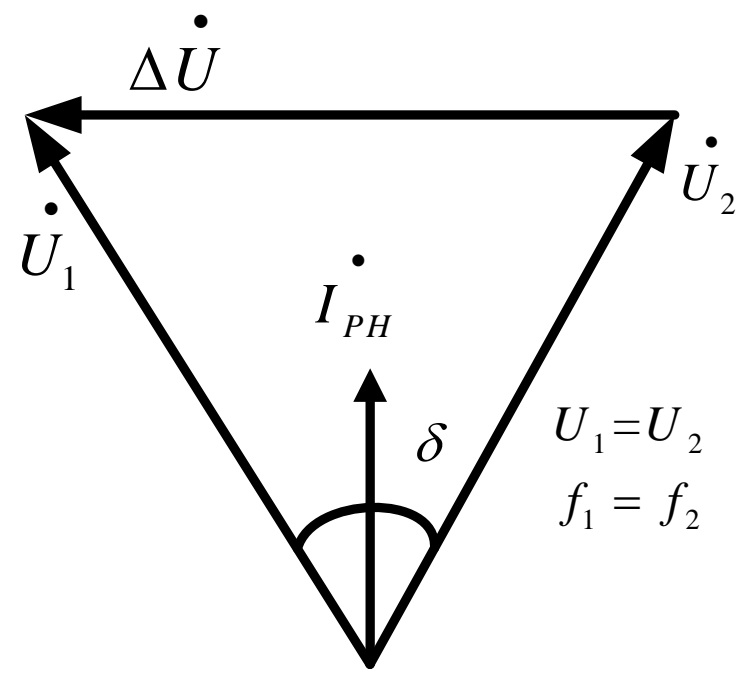

Fig. 2 the phasor diagram of $\delta_{2} \neq \delta_{1}$

(3). Fig.3 show the situation of $U_{2}=U_{1}, \delta_{2}=\delta_{1}, f_{2} \neq f_{1}$,

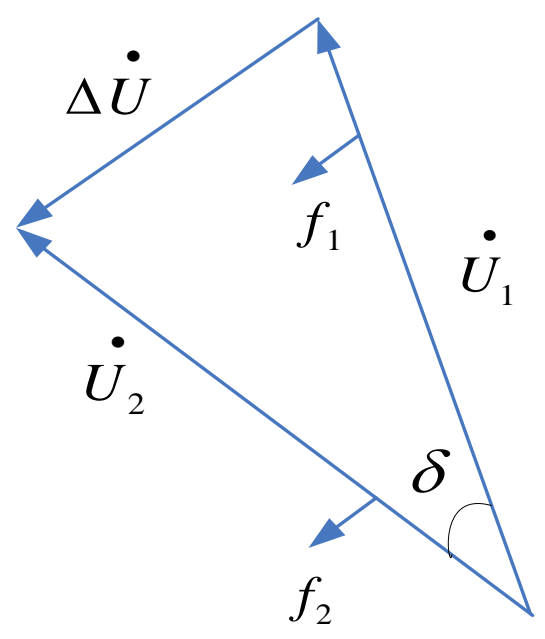

Fig.3 the phasor diagram of $f_{2} \neq f_{1}$ 


\section{The Design of Parallel Operation}

Every generating unit is completed by the control unit of the generating unit. The self- starting capability composed of some logical judgment. When the control system detects that the power of the electric network is not enough or the power is not enough, it would form an order of increasing machine immediately. At the same time, the logical condition of the start of the corresponding unit is detected. If the mechanical fault or the electric fault is detected when the logical condition is enough to start, the computer would turn to the external circuit to input signal and analyze the value of the internal flag variable, therefore, it could make a decision whether the generating unit can carry out "self-starting". After one generator carries out "self-starting”, the program detects the generator whether it is started successfully. If it is not successes, starts again. If the generator fails three times, the other one would start. After the generator starts successfully, it turns to a single frequency modulation program automatically or the system calls “automatic parallel operation” program. Moreover, the key of manual parallel operation is to detect and adjust the frequency and initial phase of two generators which are waiting for parallel operation[5].Fig.4 describes the flowchart of parallel operation.

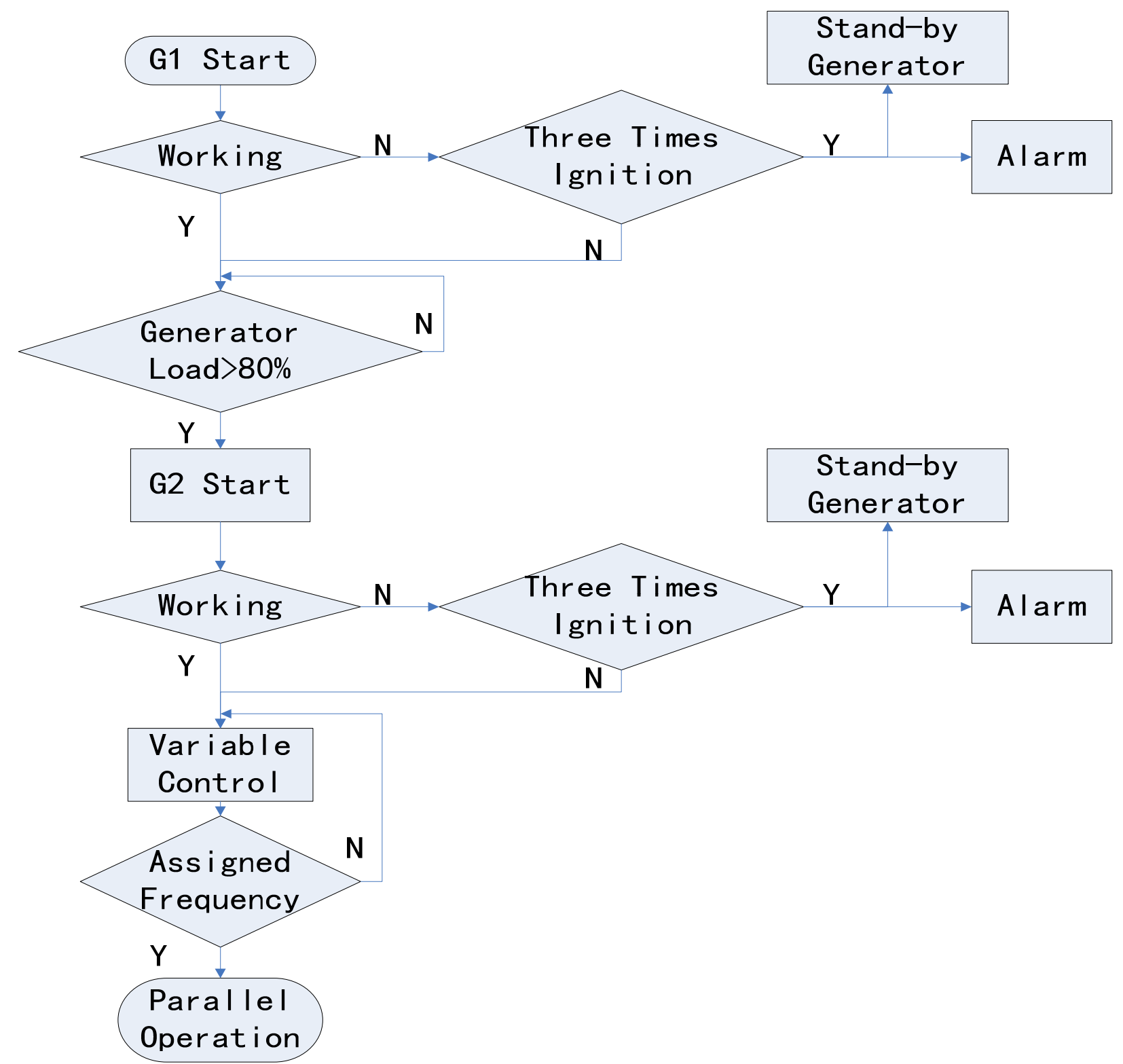

Fig.4 parallel operation flowchart 


\section{Mathematical Model for The Synchronous Generator}

The marine power station simulation is realized through operation on digital computers. The primary job is to set up a mathematical model that describes the marine power station simulation system when we develop a simulator. We'll infer a dynamic model with the Park equation derived from the $\mathrm{d}, \mathrm{q}$ coordinate system as a basic equation for the synchronous generator. In certain special cases, a simplified model can be obtained from the complete dynamic model. With little disorder, the complete non-linear model can be changed into a linear one at the condition point.Mathematical model for the synchronous generator considering the function of the damping winding,the Park equation (matrix) with a per unit value (1):

$\left[\begin{array}{l}U_{d} \\ U_{f} \\ U_{q}\end{array}\right]=\left[\begin{array}{ccc}-r_{a} & 0 & x_{q} \\ 0 & r_{q} & 0 \\ -x_{d} & x_{a d} & -r_{q}\end{array}\right] \cdot\left[\begin{array}{c}i_{d} \\ i_{f} \\ i_{q}\end{array}\right]+\left[\begin{array}{ccc}-x_{d} & x_{a d} & 0 \\ -x_{a d} & x_{f} & 0 \\ 0 & 0 & -x_{q}\end{array}\right] \cdot\left[\begin{array}{c}p i_{d} \\ p i_{f} \\ p i_{q}\end{array}\right]$

$U_{d}, U_{q}$ and $U_{f}$ : d axis voltage, q axis voltage and excitation voltage; $i_{d}, i_{q}$ and $i_{f}$ : d axis current,q axis current and excitation current; $x_{d}, x_{q}$ and $x_{f}: \mathrm{d}$ axis reactance,q axis reactance and excitation reactance; $\mathrm{p}$ : differential expression; $r_{d}, r_{f}:$ armature resistance, excitation resistance[6].

A rotor motion equation (2)(3)

$H p \omega=M m-M e-M d$

$p \delta=\omega-1$

$M m$ : mechanical torque, $M m=D_{W}$ : damping torque, $M e$ : the per unit value of the electromagnetic torque with Three phase voltage. $\delta$ :power angle, $\omega$ 错误! 未找到引用源。: angular frequency.

Static load model (4):

$\left[\begin{array}{c}p_{d}^{i} \\ p_{q}^{i}\end{array}\right]=\left[\begin{array}{cc}-\frac{r_{a}}{x_{a}} & 1 \\ -1 & -\frac{r_{q}}{x_{q}}\end{array}\right] \cdot\left[\begin{array}{c}i_{d} \\ i_{q}\end{array}\right]+\left[\begin{array}{cc}\frac{1}{x_{a}} & 0 \\ 0 & \frac{1}{x_{q}}\end{array}\right] \cdot\left[\begin{array}{l}u_{d} \\ u_{q}\end{array}\right]$

错误! 未找到引用源。 $r_{a}$ : load resistance, $x_{a}$ :load reactance

The complete dynamic model for a synchronous generator, its matrix is a state equation. (1) (4)[7].

\section{The Implemtation of Automatic Parallel Operation}

Define several variables:

int Value2; //the power of SG1

int Value3; //the frequency of SG1

int Value4; //the voltage of SG1

int Value6; // the power of SG2

int Value7; // the frequency of SG2

int Value8; // the voltage of SG2

The initial value is set to 0 . The control function realized by SetTimer(),KillTimer() and OnTimer().If the power of SG1 is over $800 \mathrm{KW}, \mathrm{SG} 2$ will start automatically.

if (Value2 $>800$ ) 


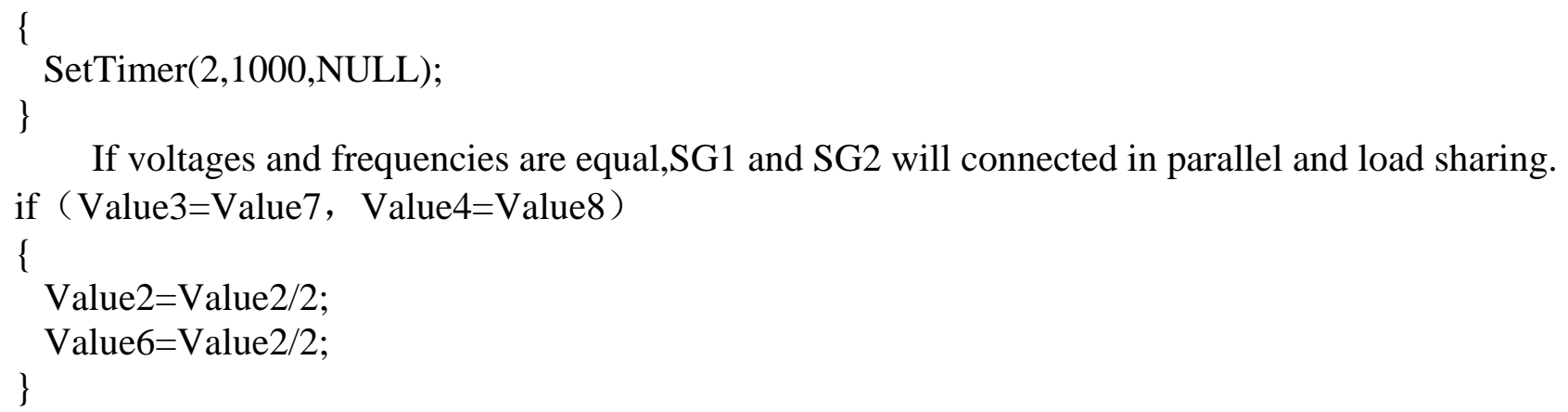

\section{Summary}

The paper investigates automatic parallel operation for marine power station simulation based on virtual technology. The design, with high reliability, powerful function and steady performance, improves power supply quality of the power system. Moreover, it is very useful for implementation of power plant automation, and it has a wide application prospect. Nowadays, marine power station system has been successfully used in various universities and has a satisfied feedback.

\section{Acknowledgements}

This work was financially supported by the Guangzhou Huangpu District Science and Technology Research Project (201237), which was supported by the construction project of strengthening universities with innovation in 2014 as well.

\section{References}

[1]Zhao Xu,Zhiliang Wu. Design of Automatic Parallel Operation Set for Marine Power Station Based on S7-1200 PLC[J].Marine Electric.Vo1.34 (2014) p. 11

[2]Yongjian Wang,Guohao Yang. Modaling for V.R. Ship's switch board and realization for simulating operation of manual paralleling.Jimei University.Vo1.30(2009)

[3]Huayao Zheng. Marine Electrical Equipment and System II. The publisher Of Dalian Maritime University.2011

[4]Erdal Bekiroglu,Alper Bayrak. AUTOMATIC SYNCHRONIZATION UNIT FOR THE PARALLEL OPERATION OF SYNCHRONOUS GENERATORS.IEEE.2009

[5]Chuang Wang. Design of Monitoring and Controlling System of Ship Power Station Based on PLC.Dalian Maritime University.May 2008

[6]Caiqin Sun, Chen Guo, Chengjun Shi. Implementation of Marine Power Station Simulation System. Dalian Maritime University.Oct,24-27, 2005

[7]Limin Zhou.Research and realize of simulator of Ship Power Station.Dalian Maritime University.March 2005 